\title{
APPLIANCE
}

\section{OPHTHALMOSCOPIC ATTACHMENT FOR THE DIAGNOSIS OF ECCENTRIC FIXATION*}

\author{
BY \\ H. H. MASSIE \\ From the Regional Eye Unit, South Middlesex Hospital, Isleworth, London
}

THERE is much need for earlier and more careful examination of patients, particularly children, who present with amblyopia in a squinting eye, with a view to the detection of eccentric fixation.

It is true that in many cases considerable improvement of visual acuity may occur as a result of occlusion of the non-squinting eye, but there are others, mainly those with eccentric fixation, in which vision remains unaffected. Indeed, occlusion of the non-squinting eye only serves to condition the eccentric fixation reflex, when this is present, as well as to increase the macular suppression. Thus treatment of the amblyopia, when it is eventually undertaken on modern lines (Mayweg and Massie, 1958), becomes a more lengthy and uneconomical procedure.

Only gross degrees of eccentric fixation can be detected by means of the cover test. Lesser degrees of eccentric fixation, especially when the fixation is peri- or para-macular, cannot be diagnosed without some special instrument such as the Visuscope, although eccentricity may be strongly suspected to be present in cases in which vision has not improved beyond $6 / 60$, or even $6 / 24$, after several months of total occlusion.

The ready availability of a simple instrument for the examination of fixation in these cases of amblyopia is desirable. It is more satisfactory for all concerned if the ophthalmologist can decide about the position of the fixation for himself before treatment is started. For this purpose a palegreen filter (No. 21 pea-green), in which there is a central uncoloured hole of $3 / 1,000$ in., may easily be fitted to most of the ophthalmoscopes in common use. This pale-green filter with the clear centre does not present too great a contrast when viewing the fundus, such as occurs when the green in the filter is too dark. On the Keeler ophthalmoscope this filter can now be supplied as an extra attachment, and it is fitted onto the rotating dome holding the different-sized apertures. A filter can easily be fitted on the A.I.M. ophthalmoscope, and it fits as a cap over the condensing lens on the Lister Morton ophthalmoscope. The white circle of light is clearly projected onto the fundus, and because of the simultaneous contrast, is easily visible

* Received for publication June 8, 1959. 
both to the patient and to the examiner. The refractive error of the patient has been found to be unimportant when this filter is used, since eccentric fixation seems to occur mainly in emmetropes or hypermetropes, and it is only in cases of myopia of $-6 \mathrm{D} \mathrm{sph}$. and greater that the white circle of light on the fundus becomes indistinct.

The ophthalmoscope, with the filter fitted as described, can be used to examine the fixation in most cases, even with undilated pupils and in children under the age of 5 years. If the light is kept on the macular area, the pupil gradually dilates and the point of fixation is easily seen. If the examiner is in any doubt about the position of the fixation, bilateral mydriasis should be employed. The patient is instructed to look directly at the bright circle of light and the examiner notes the exact position on the fundus where the light is located. In cases of eccentric fixation the central clear area may be seen anywhere between the fovea and the disc (Figure). The area of fundus actually seen is about 1.5 disc diameters. The eye not under examination must be kept firmly closed, otherwise an erroneous result will be obtained.

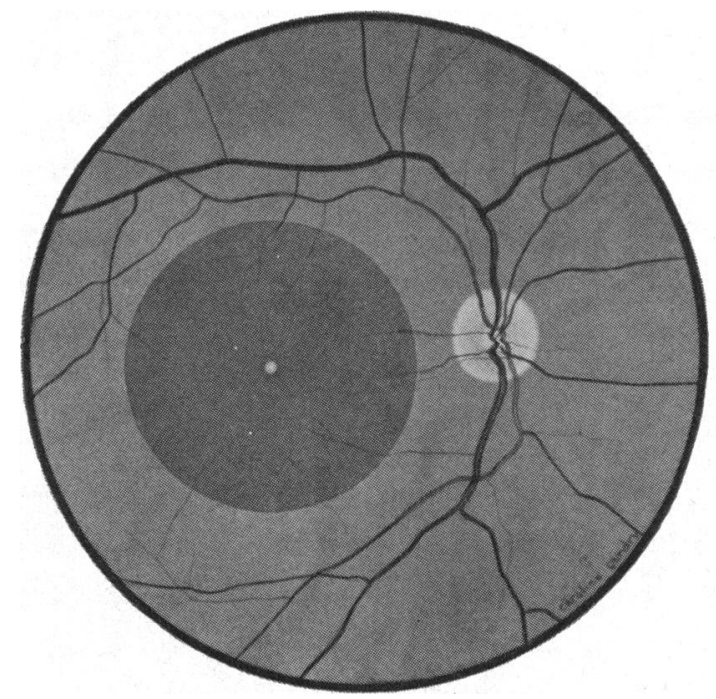

FiguRE.-Fundus showing contrast between green filter and central uncoloured area centred over the fovea.

If the diagnosis of eccentric fixation in an amblyopic eye can be made when the child is first seen, at say the age of 2 to 3 years, occlusion of the eccentrically fixing (or squinting) eye should be undertaken for a period of 6 to 8 weeks initially. The diagnosis can be made when the child's eyes are under atropine for routine estimation of the refractive error and fundus examination, even if the child is too young or too uncooperative for estimation of visual acuity. To ensure that the child understands what is required, 
the non-squinting eye is examined first. If the occluded, eccentrically-fixing eye is then examined at fortnightly intervals, the fixation will be seen to move gradually nearer to the fovea. The correct time to change the occluded eye is when the fixation becomes unsteady, on and off the macula. Occasionally the eye appears to have no fixation at all.

\section{Summary}

An attachment to the standard ophthalmoscopes is described to enable these instruments to be used for the early diagnosis of eccentric fixation in cases of amblyopia ex anopsia.

The author wishes to thank Mr. T. Keith Lyle for the helpful criticisms he has given in the preparation of this paper, and Miss Caroline Gundry for the preparation of the diagram.

The filter described has been developed by Messrs. C. Davis Keeler for use as an attachment on the Keeler "Specialist" ophthalmoscope and costs about 20 s.

\section{REFERENCE}

MAYWEG, S., and MASSIE, H. H. (1958). Brit. J. Ophthal., 42, 257. 